Revue internationale P.M.E.

Économie et gestion de la petite et moyenne entreprise

\title{
MANAGEMENT DE LA PETITE ENTREPRISE DES LOISIRS SPORTIFS : UNE APPROCHE SOCIOÉCONOMIQUE, Malek Bouhaouala, Bruxelles, De Boeck Université, 2008, 158 p.
}

\section{André Joyal}

Volume 21, numéro 3-4, 2008

État et perspectives de la recherche germanophone en entrepreneuriat et PME

URI : https://id.erudit.org/iderudit/038040ar

DOI : https://doi.org/10.7202/038040ar

Aller au sommaire du numéro

Éditeur(s)

Presses de l'Université du Québec

ISSN

0776-5436 (imprimé)

1918-9699 (numérique)

Découvrir la revue

Citer ce compte rendu

Joyal, A. (2008). Compte rendu de [MANAGEMENT DE LA PETITE ENTREPRISE DES LOISIRS SPORTIFS : UNE APPROCHE SOCIOÉCONOMIQUE, Malek

Bouhaouala, Bruxelles, De Boeck Université, 2008, 158 p.] Revue internationale P.M.E., 21(3-4), 189-193. https://doi.org/10.7202/038040ar d'utilisation que vous pouvez consulter en ligne. 


\section{MANAGEMENT DE LA PETITE ENTREPRISE DES LOISIRS SPORTIFS: UNE APPROCHE SOCIOÉCONOMIQUE Malek Bouhaouala Bruxelles, De Boeck Université, 2008, 158 p.}

Pour bien saisir l'intérêt de cet ouvrage dont l'auteur est responsable de la Maison de l'Entrepreneuriat de Grenoble Université (sic) tout en étant maître de conférences à l'Université Joseph-Fourier Grenoble I, il importe de porter attention au sous-titre. En effet, Malek Bouhaouala inscrit son analyse dans une perspective où l'entreprise et le marché se trouvent conçus comme fondamentalement socialisés, c'est-à-dire susceptibles d'être expliqués à l'aide de variables sociologiques. Ce faisant, il devient possible de fournir une alternative aux interprétations d'inspiration néoclassique que les étudiants se font servir à souhait dans tout cours d'introduction à la microéconomie 101 (genre Économie pour les nuls). Suivant l'optique privilégiée dans cet ouvrage, le marché serait un espace social d'échanges alors que, comme le précise l'auteur, l'activité économique serait l'émanation d'une diversité de logiques d'actions pratiques dépassant la rationalisation abstraite de l'homo oconomicus.

De toute évidence, les références bibliographiques en font foi,l'intérêt envers ce sujet et l'approche ici favorisée remontent à une thèse de doctorat soutenue à cette même Université de Grenoble I en 1999. En s'intéressant au secteur du tourisme et des loisirs sportifs, l'auteur a pu développer une conception humaniste de l'entreprise. Ce qui l'amène à écrire à plus d'une occasion que l'entrepreneur n'est pas exclusivement motivé par la fameuse maximisation du profit dont on gave les étudiants dans les cours d'économie depuis bientôt un siècle (merci Marshall et à ses vulgarisateurs tels les Samuelson et cie). Lors d'un colloque tenu à Dijon il y a quelques années et à la suite d'une communication d'un jeune collègue portant sur l'entrepreneuriat, je n'ai pu m'empêcher de lui signaler qu'en 20 minutes il avait utilisé l'expression «maximisation du profit» une quinzaine de fois et que cette prétendue motivation de la part de l'entrepreneur n'a rien à voir avec la réalité. Suffit d'en rencontrer quelques-uns pour s'en convaincre.

Sans mettre de préalable à la compréhension ni à l'explication du management de la petite entreprise, Malek Bouhaouala se donne pour objectif d'attirer l'attention des formateurs, des décideurs et des professionnels de l'accompagnement de l'entrepreneur-dirigeant sur quatre points importants liés au le rôle économique et social de la petite entreprise. Ce sont:

- une analyse des «managements» et des «comportements» émancipée de l'orthodoxie économique et managériale; 
- les réalités vécues par l'entrepreneur afin de confectionner des programmes de formation en entrepreneuriat;

- la nécessité pour les programmes d'aide et d'accompagnement à la création de la petite entreprise de mieux cibler leurs actions en fonction des motivations et des profils sociologiques des porteurs de projets;

- la pertinence d'introduire des variables non économiques dans l'évaluation de la performance de la petite entreprise.

C'est ce qui conduit Boualem Aliquat, de l'Université de Nice SophiaAntipolis, à relever dans la préface que l'intérêt de cet ouvrage réside non seulement dans le fait que l'on trouve très peu d'ouvrages consacrés aux entreprises ici considérées, mais aussi dans le fait que, précisément, l'auteur en présente une analyse socioéconomique, ce qui s'avère tout à fait inusité. La taxonomie retenue se rapporte à quatre types d'entreprise et d'entrepreneur, à savoir 1) la microentreprise unipersonnelle de l'Indépendant passionné; 2) l'Agence des Sports Outdoor (exit l'expression sports de plein-air... faut croire qu'elle fait trop ringard); 3) l'entreprise familiale du Conservateur patrimonial; 4) l'organisation sociomanagériale du Manager gestionnaire. L'auteur aborde les uns et les autres en refusant de concevoir l'entreprise comme une simple boîte noire, un centre de décision parfaitement rationnel assujetti aux fluctuations du marché et aux pressions de la concurrence, comme si les entrepreneurs réagissaient mécaniquement aux exigences de la loi de l'offre et de la demande. Pour chacun des quatre types d'entrepreneur étudiés, Malek Bouhaouala recourt à cinq principes organisateurs: micromentalité - forme organisationnelle - stratégies - marketing - logique d'action. Un tableau synthèse permet de les relier à cinq dimensions typologiques. En ce qui regarde l'entrepreneur Indépendant passionné, on obtient : passionnelle - microentreprise unipersonnelle implicite de spécialisation par produit-passion - démarche artisanale centrée sur le sport-passion - l'entrepreneur hédoniste. Ne pouvant tout définir, retenons que la micromentalité est un concept synthétique regroupant un ensemble non systématiquement cohérent de valeurs, de conceptions, de motifs et de buts qui contribuent à créer une vision subjective du monde chez l'entrepreneur. Un chapitre distinct est consacré à la description des quatre types d'entrepreneur que l'auteur nous aide à découvrir à travers de nombreuses citations issues de ses entrevues.

Pour l'Indépendant passionné, on l'aurait deviné, l'entreprise et le profit ne sont rien d'autre que des moyens pour vivre une passion du sport et pour vivre en accord avec son style de vie. Qu'on en juge par ces expressions recueillies par l'auteur: «choix personnel de vie», «un art de vivre», «pas 
de boss», «permet de faire ce que je veux». On retrouve ici la description que fait Michel Houellebecq de son père qui a préféré être moniteur de ski indépendant plutôt que d'adhérer à la prestigieuse Compagnie des guides de Chamonix: «Voilà un homme qui aura tout sacrifié dans sa vie, absolument tout, à ce seul impératif, ne dépendre de personne ${ }^{1}$. L'Indépendant passionné a l'avantage d'avoir des clients qui lui sont fidèles et qui parfois deviennent des amis. J'ai pu recueillir moi-même des témoignages de tels entrepreneurs néoruraux (des soixante-huitards) en Ariège.

L'entrepreneur Indépendant, quant à lui, s'il recherche à la fois la rentabilité, la pérennité, la croissance et le développement de son entreprise, ne s'inscrit pas pour autant dans l'idéal qui caractérise l'entrepreneur capitaliste classique. L'auteur voit en lui un individu doté d'un double rapport à l'argent: un signe de réussite sociale et professionnelle et un instrument économique au service de l'action entrepreneuriale. Les services sportifs qu'il commercialise se rapportent à des activités estivales et hivernales: sports aériens tel le parapente, sports aquatique tel le canyoning, sports verticaux telle l'escalade, sports terrestres comme la randonnée ou le VTT. L'obligation de présenter un visage sérieux (!) conduit cet entrepreneur à donner à son entreprise le label d'Agence en Sports Outdoor. En France: succès garanti... même si c'est un des rares mots de la langue de Shakespeare que le client peut connaître. Il peut avoir du personnel d'encadrement variant de deux à douze dans un contexte de management centralisé. Comme caractéristique de sa micromentalité, on trouve en lui une conception de l'entreprise voulant qu'elle soit un objet d'accomplissement de soi et un lieu de création. Et son orientation stratégique se rapporte au produit, à l'innovation et au client.

Pour sa part, l'entrepreneur Conservateur patrimonial se distingue par un mode de management paternaliste, déterminé par les dimensions conservatrices et patrimoniales et par une démarche productive inadéquate avec les finalités économiques et marketing. Deux caractéristiques qui, selon l'auteur, le situent à la fois dans l'idéologie du commerçant et dans celle de l'artisan. L'entreprise est pour lui un moyen de garantir son indépendance tout en conservant la cohésion familiale comme en fait foi ce témoignage: «Quand on crée son entreprise, c'est pour gagner de l'argent, mais moi ce n'est pas pour faire fortune, ce qui m'intéresse, c'est d'avoir une certaine liberté et puis je privilégie ma famille. Donc, c'est pour ça que je travaille de chez moi, que je suis toujours à la maison ». Les stratégies de cet entrepreneur

1. Michel Houllebecq et Bernard-Henry Lévy, Ennemis publics, 2008, Paris, Flammarion-Grasset, p. 67. 
sont fondées sur des réseaux formels et informels prenant appui sur des intérêts commerciaux et des liens sociaux locaux. L'ancienneté sur le marché et l'ancrage territorial constituent ses atouts stratégiques.

Le chapitre 5 consacré au Manager gestionnaire m'a réservé une surprise agréable: me voir cité à quatre reprises pour un article complètement oublié, publié en 1986 et dont la première version remonte ainsi à environ un quart de siècle. Oui, avouons-le: rien pour me rajeunir, mais c'est suffisant pour rappeler à mon souvenir une époque que les moins de 20 ans ne peuvent pas connaître (merci Aznavour). C'était au temps, non pas du cinéma muet (merci Brel), mais de l'entreprise dite alternative, ainsi définie par sa double finalité: à la fois sociale et économique, que l'on désigne aujourd'hui, au Québec, par l'expression «entreprise d'économie sociale». Dans ces pages, il y a 10 ans, j'ai fait la recension d'un ouvrage se rapportant à une entreprise on ne peut plus alternative, soit celle d'Ambiance Bois du Plateau de Millevaches, dans le «fond» de la Creuse ${ }^{2}$. Selon l'auteur, le Manager gestionnaire et l'entrepreneuriat de type alternatif ont en comun des objectifs économiques retraduits en termes d'objectifs sociaux. Cet entrepreneur, en effet, s'intéresse autant à la création d'une plus-value sociale qu'économique. Comme chez mes entrepreneurs alternatifs des années 1980, on trouve ici un individu dont la micromentalité se fonde sur des valeurs faisant référence au partage social et au dévouement. Qu'on en juge par les maîtres mots suivants qui aident à l'identifier: solidarité, responsabilité, honnêteté, engagement, utilité sociale. Et voici un témoignage que ne renieraient pas mes amis du Plateau de Millevaches: «On est des privilégiés par rapport aux gens qui sont sur les périphériques tous les matins dans leur voiture. C'est vrai qu'en venant de la ville pour travailler ici j'ai perdu en salaire, mais je gagne en qualité de vie.» Les exemples donnés ici ne manquent pas de me rappeler des souvenirs: le tourisme pour handicapés (au Québec, aujourd'hui, la rectitude politique envahissante oblige à écrire: personnes handicapées), le tourisme vert, l'écotourisme, le tourisme éducatif, le développement local par les loisir ( $c f$. nos bases plein-air pour familles) et le tourisme sportif. Cependant, je dois apporter un bémol à l'interprétation de notre collègue grenoblois. Comme il arrive souvent lorsqu'on est cité, on aurait préféré que l'auteur se rapporte à une autre publication, sur le même sujet, que l'on estime d'intérêt supérieur à celle mentionnée. Ainsi, de toute évidence, l'auteur ne connaît pas l'ouvrage publié en 1987 en collaboration avec un collègue de l'Université Laval intitulé: L'entreprise alternative : entre mirages et réalité. $\mathrm{Il}$ aurait pu voir que notre entrepreneur alternatif, contrairement à ce qu'il décrit dans un encadré sur les caractéristiques organisationnelles, ne pratique en rien une direction hiérarchisée selon les orientations du

2. Cf. vol 12, no 4,1999, p. 103 . 
conseil d'administration. Bien au contraire, l'autogestion fut l'une des quatre dimensions retenues pour définir une entreprise alternative. Pour dire vrai, l'entrepreneur alternatif étudié il y a des lustres, par ses motivations et son comportement, me paraît plus près du Passionné indépendant. Qu'on en juge par ce que l'auteur écrit dans un court avant-dernier chapitre où il traite de l'influence de la micromentalité sur les choix et stratégies de la petite entreprise: le type d'organisation dirigée par le Manager gestionnaire se distingue par 1) une plus forte division du travail et la spécialisation des personnels salariés , 2) une diversification horizontale des prestations et 3) un mode de gestion managérial hiérarchisé. On s'éloigne alors de façon sensible de la vision dégagée de l'entrepreneur alternatif qui définissait son entreprise comme un lieu d'expérimentation sociale. Mais ce n'est là,je le répète, qu'un bémol dans un ensemble qui, par ailleurs, tient bien la route.

L'ouvrage se termine par un chapitre intitulé «L'offre locale socialisée ». On y trouve une intéressante allusion au territoire vu comme une entité qui prend la dimension que lui confèrent les acteurs socioéconomiques qui le constituent, ainsi que l'expression identitaire qu'il suscite chez eux. L'auteur en arrive ainsi à considérer le marché territorialisé en termes d'offre locale socialisée constituée d'un croisement de liens territoriaux et professionnels tout en traitant les questions de la concurrence entre les différents types d'entreprises.

L'ouvrage, rédigé dans un style élégant, est fort plaisant à lire. En outre, une présentation aérée, accompagnée de plusieurs tableaux ou encadrés synthèses, met bien en relief ce qui caractérise les quatre types d'entrepreneurs considérés.

André Joyal

Institut de recherche sur les $P M E$

Université du Québec à Trois-Rivières 